\title{
IVD.09 - Development of an identification system of Neisseria meningitidis, Streptococcus pneumoniae and Haemophilus influenzae in clinical samples, by qPCR-HRM
}

Ivano de Filippis ${ }^{1 *}$; Claudia Ferreira de Andrade ${ }^{1}$; Aline Carvalho de Azevedo $^{2}$; Antonio Eugenio C. C. de Almeida ${ }^{1}$.

1 Fiocruz/INCQS;

\section{Fiocruz/INCQS/PPGVS}

Introduction: The three main etiological agents of bacterial meningitis (N. meningitidis - Nm, S. pneumoniae - Sp and $\mathrm{H}$. influenzae - Hi) are characterized as fastidious bacteria for detection in clinical samples by conventional laboratory methods. They cause acute disease with rapid evolution and high lethality rates and sequelae. Rapid diagnosis of these agents is extremely important for management of patients, and better prognosis. Several molecular diagnostic methods based on the polymerase chain reaction (PCR) have been developed for the detection of these pathogens such as endpoint PCR or Real-Time PCR (qPCR) where the Taqman system is the most used. Both methods include the same reaction dynamics, but in the Taqman system a fluorescent probe detects the presence of the target in the sample making the tests more expensive. The proposal of this study is the use of a method called High Resolution Melting (HRM) with the same sensitivity of qPCR-Taqman, but at a lower cost since it does not use probes.

Objective: The aim of this project is to design and optimize a multiplex reaction that can detect one of the three pathogens by one-step reaction from a clinical sample (blood, sera or CSF) using the qPCR-HRM system. After optimization, the system will be developed as a kit for use in the Public Health System.

Methodology: Reference strains of the three pathogens with different serogroups and serotypes were used as positive controls. Other bacterial species normally isolated from invasive infections causing similar symptoms, were also tested as negative controls. We used Eva Green as DNA fluorescent dye during the amplification reactions. Three different exclusive gene targets for each specie were used to detect the three pathogens (nspA-Nm, ply-Sp and P6-Hi).The system has been tested against reference strains, clinical isolates strains and clinical samples with negative results after conventional laboratory tests.

Results: The qPCR-HRM test showed three specific and distinct TM for each positive target. All reference $\mathrm{Nm}$, Sp and Hi strains from any serogroup/serotypes, gave positive amplification with their specific TM. No amplification was detected from other bacterial species different from the three target pathogens. At least 30\% of negative samples after conventional laboratory tests, showed amplification for one of the three agents. Limit of Detection for each target was established at 200fg/ $\mathrm{\mu l}$.

Conclusions: The proposed diagnostic system using qPCR-HRM showed to be as sensitive and specific as the Taqman system and more sensitive than conventional laboratory tests. After a rough calculation, we believe that the costs/sample using the qPCR-HRM system can be about $22 \%$ of the cost/sample using the Taqman system. Our goal is that the qPCR-HRM could be used as a kit for bacterial meningitis diagnostic in the public Health System. Patent deposit \#BR102018003245-3 accepted on 20/02/2018.

Conclusion: The proposed diagnostic system using qPCR-HRM, showed to be as sensitive and specific as the Taqman system and more sensitive than conventional laboratory tests. After a rough calculation, we believe that the costs/sample using the qPCR-HRM system can be about $22 \%$ of the cost/sample using the Taqman system. Our goal is that the qPCR-HRM could be used as a kit for bacterial meningitis diagnostic in the public Health System. Patent deposit \#BR102018003245-3 accepted on 20/02/2018.

Keywords: Bacterial meningitis; qPCR-HRM; rapid diagnostic 